\title{
Fixed-Point Theorems in Fuzzy Metric Spaces via Fuzzy F-Contraction
}

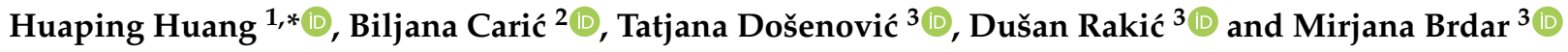 \\ 1 School of Mathematics and Statistics, Chongqing Three Gorges University, Wanzhou 404020, China \\ 2 Faculty of Technical Sciences, University of Novi Sad, Trg Dositeja Obradovića 6, 21000 Novi Sad, Serbia; \\ biljana@uns.ac.rs \\ 3 Faculty of Technology Novi Sad, University of Novi Sad, Bulevar cara Lazara 1, 21000 Novi Sad, Serbia; \\ tatjanad@uns.ac.rs (T.D.); dusan.rakic@uns.ac.rs (D.R.); mirjana.brdar@uns.ac.rs (M.B.) \\ * Correspondence: huaping@sanxiau.edu.cn
}

Citation: Huang, H.; Carić, B.;

Došenović, T.; Rakić, D.; Brdar, M.

Fixed-Point Theorems in Fuzzy

Metric Spaces via Fuzzy

F-Contraction. Mathematics 2021, 9,

641. https://doi.org/

10.3390/math9060641

Academic Editor: Antonio Francisco Roldán López de Hierro

Received: 27 January 2021

Accepted: 15 March 2021

Published: 17 March 2021

Publisher's Note: MDPI stays neutral with regard to jurisdictional claims in published maps and institutional affiliations.

Copyright: (c) 2021 by the authors. Licensee MDPI, Basel, Switzerland. This article is an open access article distributed under the terms and conditions of the Creative Commons Attribution (CC BY) license (https:/ / creativecommons.org/licenses/by/ $4.0 /)$.

\begin{abstract}
The purpose of this paper is to introduce a new type of contraction called fuzzy $F$ contraction. As compared to the F-contraction in the existing literature, our fuzzy F-contraction is much simpler and more straightforward, since it contains only one condition-that is, the function $F$ is strictly increasing. Moreover, some fixed-point theorems for fuzzy F-contraction are presented. Further, some examples are given to illustrate its validity and superiority. In addition, by applying a very significant lemma, we show that our proofs of most fixed-point theorems are shorter and more elegant than ones in the literature.
\end{abstract}

Keywords: fixed-point; fuzzy metric space; $t$-norm; fuzzy $F$-contraction

\section{Introduction and Preliminaries}

Based on the theory of fuzzy sets introduced by Zadeh [1], George and Veeramani [2], provided axioms to fuzzy metric spaces. One of the most influential axioms regarding binary functions is the triangular norm (for short, $t$-norm), which first appeared in the work of Schweizer and Sklar [3]. This is a crucial operation in several fields, such as fuzzy sets, fuzzy logic, and their applications.

Starting with the famous Banach contraction principle [4], a large number of mathematicians started to formulate various contractive conditions under which a fixed point exists. One of the most interesting motivations is the fixed-point theory established in fuzzy metric spaces, which was initiated by Grabiec [5], where a fuzzy metric version of the Banach contraction principle was presented. Subsequently, Gregori and his coauthors introduced a variety of fuzzy contractive mappings in fuzzy metric spaces (see [6-8]). On the other hand, Mihet $[9,10]$ proposed a fixed-point theorem for weak Banach contraction in $W$-complete fuzzy metric spaces and generalized the previous results involving some new type of contractions, such as Edelstein fuzzy contractive mappings, fuzzy $\psi$-contractive mappings, and so forth (for details, see [11]). Recently, Wardowski [12] introduced a new concept of fuzzy $H$-contractive mapping and obtained some relevant fixed-point theorems. Additionally, Wardowski [13] introduced a contraction called F-contraction and proved a fixed-point theorem in metric spaces. Very recently, [11,14] gave other contractions in fuzzy metric spaces.

Throughout this paper, we introduce a new contraction called fuzzy F-contraction, which is different from that of $[12,15]$ since our contraction contains a simpler condition, that is, the mapping is only strictly increasing. Moreover, we cope with fixed-point theorems for fuzzy F-contraction in the setting of fuzzy metric spaces. Specifically, we first give a lemma with respect to the Cauchy sequence in fuzzy metric spaces. Second, we introduce the concept of fuzzy F-contraction, in which the function only needs a strictly increasing condition. Third, utilizing the lemma mentioned above, we obtain some fixed-point theorems for fuzzy F-contraction with shorter conditions and straightforward proofs. Fourth, 
we present some examples to support our results. Our examples show that our conclusions are indeed the real generalizations in the existing literature.

In what follows, we recall several basic definitions and concepts.

Definition 1 ([3]). A binary operation $T:[0,1] \times[0,1] \rightarrow[0,1]$ is called a triangular norm (for short, $t$-norm) if the following conditions hold:

(i) $T(\gamma, 1)=\gamma$, for each $\gamma \in[0,1]$;

(ii) $T(\gamma, \delta) \leq T(\lambda, \mu)$, for any $\gamma \leq \lambda, \delta \leq \mu$ and $\gamma, \delta, \lambda, \mu \in[0,1]$;

(iii) $T$ is associative and commutative.

Three basic examples of continuous $t$-norms are as follows: $T_{\min }(\gamma, \delta)=\min \{\gamma, \delta\}$, $T_{P}(\gamma, \delta)=\gamma \cdot \delta$ and $T_{L}(\gamma, \delta)=\max \{\gamma+\delta-1,0\}$ (minimum, product, and Lukasiewicz t-norm, respectively).

Definition 2 ([2]). A triple $(X, W, T)$ is called a fuzzy metric space if $X$ is a nonempty set, $T$ is a continuous $t$-norm, and $W: X^{2} \times(0,+\infty) \rightarrow[0,1]$ is a fuzzy set satisfying the following conditions:

(GV1) $W(q, r, t)>0$ for all $q, r \in X$ and $t>0$;

(GV2) $W(q, r, t)=1(t>0)$ if and only if $q=r$;

(GV3) $W(q, r, t)=W(r, q, t)$ for all $q, r \in X$ and $t>0$;

(GV4) $W(q, l, t+s) \geq T(W(q, r, t), W(r, l, s))$ for all $q, r, l \in X$ and $t, s>0$;

$(G V 5) W(q, r, \cdot):(0,+\infty) \rightarrow[0,1]$ is continuous for all $q, r \in X$.

If (GV4) is replaced by the following condition:

$(G V 4)^{\prime} W(q, l, t) \geq T(W(q, r, t), W(r, l, t))$ for all $q, r, l \in X$ and $t>0$, then $(X, W, T)$ is called a strong fuzzy metric space (see [16]).

Moreover, if $(X, W, T)$ is a fuzzy metric space, then $W$ is a continuous function on $X \times X \times(0,+\infty)($ see [17]) and $W(q, r, \cdot)$ is nondecreasing for all $q, r \in X$ (see [5]).

In the sequel, unless there is a special explanation, we always denote by $\mathbb{N}$, the set of all positive integers; $\mathbb{N}_{0}$, the set of all nonnegative integers; $\mathbb{R}$, the set of all real numbers; and $\mathbb{R}_{+}$, the set of all positive real numbers.

Definition 3 ([2]). Let $(X, W, T)$ be a fuzzy metric space and $\left\{q_{n}\right\}_{n \in \mathbb{N}}$ be a sequence in $X$. Then, we say the following:

(i) $\left\{q_{n}\right\}_{n \in \mathbb{N}}$ converges to $q \in X\left(\right.$ say $\left.\lim _{n \rightarrow \infty} q_{n}=q\right)$, if $\lim _{n \rightarrow \infty} W\left(q_{n}, q, t\right)=1$ for any $t>0$;

(ii) $\left\{q_{n}\right\}_{n \in \mathbb{N}}$ is a Cauchy sequence if, for any $\varepsilon \in(0,1)$ and $t>0$, there exists $n_{0} \in \mathbb{N}$ such that $W\left(q_{m}, q_{n}, t\right)>1-\varepsilon$ for any $m, n \geq n_{0}$;

(iii) $(X, W, T)$ is complete if every Cauchy sequence is convergent.

Definition 4 ([8]). Let $(X, W, T)$ be a fuzzy metric space and $\phi: X \rightarrow X$ a mapping. Then $\phi$ is called a Gregori and Sapena fuzzy contraction if there exists $k \in(0,1)$ such that

$$
\frac{1}{W(\phi(q), \phi(r), t)}-1 \leq k\left(\frac{1}{W(q, r, t)}-1\right)
$$

for all $q, r \in X$ and $t>0$. In this case, $k$ is called the contractive constant of $\phi$.

We say that the mapping $T: X \rightarrow X$ is called a Tirado contraction if there exists $k \in(0,1)$ such that

$$
1-W(T q, T r, t) \leq k(1-W(q, r, t))
$$

for all $q, r \in X$ and $t>0$ (see [18]). 


\section{Main Results}

Stimulated by Lemma 4 from [19], we give the following lemma in the framework of fuzzy metric spaces. It will be vital to prove our main theorems.

Lemma 1. Let $(X, W, T)$ be a fuzzy metric space and $\left\{q_{n}\right\}$ be a sequence in $X$ such that for each $n \in \mathbb{N}$,

$$
\lim _{t \rightarrow 0^{+}} W\left(q_{n}, q_{n+1}, t\right)>0,
$$

and for any $t>0$,

$$
\lim _{n \rightarrow \infty} W\left(q_{n}, q_{n+1}, t\right)=1 .
$$

If $\left\{q_{n}\right\}$ is not a Cauchy sequence in $X$, then there exist $\varepsilon \in(0,1), t_{0}>0$, and two sequences of positive integers $\left\{n_{k}\right\},\left\{m_{k}\right\}, n_{k}>m_{k}>k, k \in \mathbb{N}$, such that the following sequences

$$
\begin{gathered}
\left\{W\left(q_{m_{k}}, q_{n_{k}}, t_{0}\right)\right\},\left\{W\left(q_{m_{k}}, q_{n_{k}+1}, t_{0}\right)\right\},\left\{W\left(q_{m_{k}-1}, q_{n_{k}}, t_{0}\right)\right\}, \\
\left\{W\left(q_{m_{k}-1}, q_{n_{k}+1}, t_{0}\right)\right\},\left\{W\left(q_{m_{k}+1}, q_{n_{k}+1}, t_{0}\right)\right\}
\end{gathered}
$$

tend to $1-\varepsilon$ as $k \rightarrow \infty$.

Proof. Let $\left\{q_{n}\right\}$ be a sequence in $X$, which is not a Cauchy sequence. Then, by Definition 3, there exist $\varepsilon \in(0,1), t_{0}>0$ and sequences $\left\{n_{k}\right\}$ and $\left\{m_{k}\right\}, m_{k}>n_{k}>k$ such that for any $k \in \mathbb{N}$, we have

$$
W\left(q_{m_{k}}, q_{n_{k}}, t_{0}\right) \leq 1-\varepsilon
$$

and

$$
W\left(q_{m_{k}-1}, q_{n_{k}}, t_{0}\right)>1-\varepsilon
$$

Clearly, by (5), one has

$$
\limsup _{k \rightarrow \infty} W\left(q_{m_{k}}, q_{n_{k}}, t_{0}\right) \leq 1-\varepsilon .
$$

Using Condition (GV4), for any $k \in \mathbb{N}$ and $p \in\left(0, t_{0}\right)$, it is not hard to verify that

$$
W\left(q_{m_{k}}, q_{n_{k}}, t_{0}\right) \geq T\left(W\left(q_{m_{k}}, q_{m_{k}-1}, p\right), W\left(q_{m_{k}-1}, q_{n_{k}}, t_{0}-p\right)\right) .
$$

Note that, by (3) and (4), it follows that

$$
\lim _{n \rightarrow \infty}\left(\lim _{p \rightarrow 0^{+}} W\left(q_{n}, q_{n+1}, p\right)\right)=1 .
$$

If we take $p \rightarrow 0^{+}$in (8), then by (9), (6) and the continuity of $T$, we obtain

$$
\begin{aligned}
& \lim _{k \rightarrow \infty} W\left(q_{m_{k}}, q_{n_{k}}, t_{0}\right) \\
\geq & \lim _{k \rightarrow \infty}\left(\lim _{p \rightarrow 0^{+}} T\left(W\left(q_{m_{k}}, q_{m_{k}-1}, p\right), W\left(q_{m_{k}-1}, q_{n_{k}}, t_{0}-p\right)\right)\right) \\
= & T\left(\lim _{k \rightarrow \infty}\left(\lim _{p \rightarrow 0^{+}} W\left(q_{m_{k}}, q_{m_{k}}-1, p\right)\right), \lim _{k \rightarrow \infty}\left(\lim _{p \rightarrow 0^{+}} W\left(q_{m_{k}-1}, q_{n_{k}}, t_{0}-p\right)\right)\right) \\
= & T\left(1, \lim _{k \rightarrow \infty} W\left(q_{m_{k}}-1, q_{n_{k}}, t_{0}\right)\right) \\
= & \lim _{k \rightarrow \infty} W\left(q_{m_{k}-1}, q_{n_{k}}, t_{0}\right) \\
\geq & 1-\varepsilon .
\end{aligned}
$$


This inequality and (7) imply

$$
\lim _{k \rightarrow \infty} W\left(q_{m_{k}}, q_{n_{k}}, t_{0}\right)=1-\varepsilon
$$

Let us prove that

$$
\lim _{k \rightarrow \infty} W\left(q_{m_{k}}, q_{n_{k}+1}, t_{0}\right)=1-\varepsilon .
$$

Indeed, by (9) and (10), on the one hand, we have

$$
\begin{aligned}
& \lim _{k \rightarrow \infty} W\left(q_{m_{k}}, q_{n_{k}+1}, t_{0}\right) \\
\geq & \lim _{k \rightarrow \infty}\left(\lim _{p \rightarrow 0^{+}} T\left(W\left(q_{m_{k}}, q_{n_{k}}, t_{0}-p\right), W\left(q_{n_{k}}, q_{n_{k}+1}, p\right)\right)\right) \\
\geq & T\left(\lim _{k \rightarrow \infty} W\left(q_{m_{k}}, q_{n_{k}}, t_{0}\right), 1\right) \\
= & \lim _{k \rightarrow \infty} W\left(q_{m_{k}}, q_{n_{k}}, t_{0}\right) \\
= & 1-\varepsilon .
\end{aligned}
$$

On the other hand, by (10) and (4), we have

$$
\begin{aligned}
1-\varepsilon & =\lim _{k \rightarrow \infty} W\left(q_{m_{k}}, q_{n_{k}}, t_{0}\right) \\
& \geq \lim _{k \rightarrow \infty}\left(\lim _{p \rightarrow 0^{+}} T\left(W\left(q_{m_{k}}, q_{n_{k}+1}, t_{0}-p\right), W\left(q_{n_{k}+1}, q_{n_{k}}, p\right)\right)\right) \\
& \geq T\left(\lim _{k \rightarrow \infty} W\left(q_{m_{k}}, q_{n_{k}+1}, t_{0}\right), 1\right)=\lim _{k \rightarrow \infty} W\left(q_{m_{k}}, q_{n_{k}+1}, t_{0}\right) .
\end{aligned}
$$

Then, by (12) and (13), we obtain (11).

The left proofs are similar to the above argument, and therefore we omit them.

Remark 1. Condition (3) in Lemma 1 can be omitted if $(X, W, T)$ is a strong fuzzy metric space. In this case, instead of (8), we have

$$
W\left(q_{m_{k}}, q_{n_{k}}, t_{0}\right) \geq T\left(W\left(q_{m_{k}}, q_{m_{k}-1}, t_{0}\right), W\left(q_{m_{k}-1}, q_{n_{k}}, t_{0}\right)\right) .
$$

In the following, denote by $\mathcal{F}$ the class of all mappings $F:[0,1] \rightarrow(0,+\infty)$ satisfying the following condition: for all $q, r \in[0,1], q<r$ implies $F(q)<F(r)$. That is to say, $F$ is strictly increasing on $[0,1]$.

First of all, for the sake of the reader, we recall Definition 2.1 from [13] as follows:

Definition 5 ([13]). Let $(X, d)$ be a metric space and $F: \mathbb{R}_{+} \rightarrow \mathbb{R}$ be a mapping, satisfying the following:

$\left(F_{1}\right)$ F is strictly increasing on $\mathbb{R}_{+}$;

$\left(F_{2}\right)$ For each sequence $\left\{\alpha_{n}\right\}_{n \in \mathbb{N}}$ of positive numbers, $\lim _{n \rightarrow \infty} F\left(\alpha_{n}\right)=-\infty$ if, and only if $\lim _{n \rightarrow \infty} \alpha_{n}=0$;

$\left(F_{3}\right)$ There exists $k \in(0,1)$ such that $\lim _{\alpha \rightarrow 0^{+}} \alpha^{k} F(\alpha)=0$.

The mapping $\varphi: X \rightarrow X$ is said to be an F-contraction if there exists $\tau>0$ such that

$$
\tau+F(d(\phi(q), \phi(r))) \leq F(d(q, r))
$$

for all $q, r \in X$ with $d(\varphi(q), \varphi(r))>0$. 
Motivated by Definition 5, Definition 2.1 of [20] and Theorem 3.1 of [21], we introduce the concept of fuzzy F-contraction and prove several fixed-point theorems in fuzzy metric spaces.

Definition 6. Let $(X, W, T)$ be a fuzzy metric space and $F \in \mathcal{F}$. The mapping $\phi: X \rightarrow X$ is said to be a fuzzy F-contraction if there exists $\tau \in(0,1)$ such that

$$
\tau \cdot F(W(\phi(q), \phi(r), t)) \geq F(W(q, r, t))
$$

for all $q, r \in X, q \neq r$, and $t>0$.

Remark 2. Compared with Definition 5, Definition 6 has less conditions, since fuzzy F-contraction only requires that the mapping $F$ strictly increases, but the F-contraction has too many conditions for mapping $F$. Moreover, the condition $d(\varphi(q), \varphi(r))>0$ from Definition 5 is stronger than the condition $q \neq r$ from Definition 6 . This is because $d(\varphi(q), \varphi(r))>0$ implies $q \neq r$. Indeed, let $d(\varphi(q), \varphi(r))>0$ but $q=r$, then $\varphi(q)=\varphi(r)$. Thus, $d(\varphi(q), \varphi(r))=0$. This is $a$ contradiction. In addition, fuzzy metric spaces are more general than metric spaces. Therefore, as applications, fuzzy F-contraction is more convenient than F-contraction.

Theorem 1. Let $(X, W, T)$ be a complete fuzzy metric space such that

$$
\lim _{t \rightarrow 0^{+}} W(q, r, t)>0
$$

for all $q, r \in X$. If $\phi: X \rightarrow X$ is a continuous fuzzy F-contraction, then $\phi$ has a unique fixed-point in $X$.

Proof. Choose $q_{0} \in X$ and $q_{n+1}=\phi\left(q_{n}\right)$ for all $n \in \mathbb{N}_{0}$. Suppose that $\phi: X \rightarrow X$ is a fuzzy $F$-contractive mapping. If $q_{n}=q_{n+1}=\phi\left(q_{n}\right)$ holds for some $n \in \mathbb{N}_{0}$, then $q_{n}$ is a fixed point. Assume that $q_{n} \neq q_{n+1}$ for any $n \in \mathbb{N}_{0}$. By (14), for every $n \in \mathbb{N}$ and $t>0$, one has

$$
F\left(W\left(q_{n+1}, q_{n}, t\right)\right)>\tau \cdot F\left(W\left(q_{n+1}, q_{n}, t\right)\right) \geq F\left(W\left(q_{n}, q_{n-1}, t\right)\right) .
$$

Then, we get

$$
W\left(q_{n}, q_{n-1}, t\right)<W\left(q_{n+1}, q_{n}, t\right)
$$

Thus, $\left\{W\left(q_{n}, q_{n+1}, t\right)\right\}(t>0)$ is a strictly increasing sequence bounded from above, so $\left\{W\left(q_{n}, q_{n+1}, t\right)\right\}(t>0)$ is convergent. In other words, there exists $a(t) \in[0,1]$ such that for any $t>0$, one has

$$
\lim _{n \rightarrow \infty} W\left(q_{n}, q_{n+1}, t\right)=a(t)
$$

Clearly, for any $t>0$ and $n \in \mathbb{N}$, it follows that

$$
W\left(q_{n}, q_{n+1}, t\right)<a(t) .
$$

Note that, by (15) and (16), for any $t>0$, we have

$$
\lim _{n \rightarrow \infty} F\left(W\left(q_{n}, q_{n+1}, t\right)\right)=F(a(t)-0) .
$$

Assume that $a(t)<1$ for some $t>0$. By (14), it implies that

$$
F\left(W\left(q_{n+1}, q_{n}, t\right)\right)>\tau \cdot F\left(W\left(q_{n+1}, q_{n}, t\right)\right) \geq F\left(W\left(q_{n}, q_{n-1}, t\right)\right) .
$$

Taking the limit from both sides of (18) together with (17), we get

$$
F(a(t)-0) \geq \tau \cdot F(a(t)-0) \geq F(a(t)-0),
$$


which means that $F(a(t)-0)=0$. This is a contradiction with $F(a(t)-0)>0$. Therefore, we have

$$
\lim _{n \rightarrow \infty} W\left(q_{n}, q_{n+1}, t\right)=1 .
$$

Further, we need to prove that $\left\{q_{n}\right\}$ is a Cauchy sequence. Suppose that this claim is not true. Using Lemma 1 and noting that (19) is in fact Condition (4), then there exist $\varepsilon \in(0,1), t_{0}>0$ and sequences $\left\{q_{m_{k}}\right\}$ and $\left\{q_{n_{k}}\right\}$ such that

$$
\lim _{k \rightarrow \infty} W\left(q_{m_{k}}, q_{n_{k}}, t_{0}\right)=1-\varepsilon .
$$

By (14), we have

$$
F\left(W\left(\phi\left(q_{m_{k}}\right), \phi\left(q_{n_{k}}\right), t_{0}\right)\right)>\tau \cdot F\left(W\left(\phi\left(q_{m_{k}}\right), \phi\left(q_{n_{k}}\right), t_{0}\right)\right) \geq F\left(W\left(q_{m_{k}}, q_{n_{k}}, t_{0}\right)\right) .
$$

Letting $k \rightarrow \infty$ from both sides of the above inequality, we have

$$
F((1-\varepsilon)-0) \geq \tau \cdot F((1-\varepsilon)-0) \geq F((1-\varepsilon)-0),
$$

which establishes that $F((1-\varepsilon)-0)=0$. This is in contradiction with $F((1-\varepsilon)-0)>0$. Hence, $\left\{q_{n}\right\}$ is a Cauchy sequence. Since $(X, W, T)$ is complete, then there exists $q^{*} \in X$ such that

$$
\lim _{n \rightarrow \infty} q_{n}=q^{*} .
$$

Let us prove that $q^{*}$ is a fixed point of $\phi$. As a matter of fact, it follows immediately from (20) and the continuity of $\phi$ that

$$
q^{*}=\lim _{n \rightarrow \infty} q_{n+1}=\phi\left(\lim _{n \rightarrow \infty} q_{n}\right)=\phi\left(q^{*}\right) .
$$

Finally, we prove the uniqueness of the fixed point. Suppose that $q^{*}$ and $r^{*}$ are distinct fixed points of $\phi$. Again, by using (14), we easily obtain that

$$
F\left(W\left(\phi\left(q^{*}\right), \phi\left(r^{*}\right), t\right)\right)>\tau \cdot F\left(W\left(\phi\left(q^{*}\right), \phi\left(r^{*}\right), t\right)\right) \geq F\left(W\left(q^{*}, r^{*}, t\right)\right) .
$$

As a consequence, we have

$$
W\left(\phi\left(q^{*}\right), \phi\left(r^{*}\right), t\right)>W\left(q^{*}, r^{*}, t\right)=W\left(\phi\left(q^{*}\right), \phi\left(r^{*}\right), t\right) .
$$

This is a contradiction.

Remark 3. Let $(X, W, T)$ be a fuzzy metric space.

(i) Define a strictly increasing function $F(t)=\frac{t}{1-t}$ for any $t \in(0,1)$ and let $\phi$ be a fuzzy $F$-contraction. Then, the Gregori and Sapena fuzzy contraction (1) is obtained. Indeed, since $\phi$ is fuzzy F-contractive, then there exists $\tau \in(0,1)$ such that

$$
\tau \cdot \frac{W(\phi(q), \phi(r), t)}{1-W(\phi(q), \phi(r), t)} \geq \frac{W(q, r, t)}{1-W(q, r, t)}
$$

that is,

$$
\frac{1-W(\phi(q), \phi(r), t)}{\tau \cdot W(\phi(q), \phi(r), t)} \leq \frac{1-W(q, r, t)}{W(q, r, t)}
$$

Therefore,

$$
\frac{1}{W(\phi(q), \phi(r), t)}-1 \leq \tau\left(\frac{1}{W(q, r, t)}-1\right)
$$


holds for all $q, r \in X$, and $t>0$.

(ii) Let $F(t)=\frac{1}{1-t}$, where $t \in(0,1)$, and suppose that $\phi$ is a fuzzy F-contraction. Then we easily obtain the Tirado contraction (2).

Corollary 1. Let $(X, d)$ be a complete fuzzy metric space, and $\phi: X \rightarrow X$ be a function such that there exists $K_{i} \in(0,1)(i=1,2,3,4)$ and for all $q, r \in X, q \neq r$, and one of the following conditions holds:

(1) $K_{1} \cdot W(\phi(q), \phi(r), t) \geq W(q, r, t)$;

(2) $K_{2} \cdot \frac{1}{1+\exp \{-W(\phi(q), \phi(r), t)\}} \geq \frac{1}{1+\exp \{-W(q, r, t)\}}$;

(3) $K_{3} \cdot \frac{1}{3+\exp \{-W(\phi(q), \phi(r), t)\}-\exp \{W(\phi(q), \phi(r), t)\}} \geq \frac{1}{3+\exp \{-W(q, r, t)\}-\exp \{W(q, r, t)\}}$;

(4) $K_{4} \cdot \exp \{W(\phi(q), \phi(r), t)\} \geq \exp \{W(q, r, t)\}$.

Then, $\phi$ has a unique fixed point in $X$.

Proof. For Cases (1)-(4), put

$$
F_{1}(t)=t, \quad F_{2}(t)=\frac{1}{1+\exp \{-t\}}, \quad F_{3}(t)=\frac{1}{3+\exp \{-t\}-\exp \{t\}}, \quad F_{4}(t)=\exp \{t\} ，
$$

respectively. Using Theorem 1, we claim that $\phi$ has a unique fixed point.

In the following, we give a supportive example to illustrate Theorem 1.

Example 1. Let $X=\mathbb{R}$ and define the usual metric $d(q, r)=|q-r|$ for all $q, r \in X$. Let $T$ be a product t-norm. Define a fuzzy set as follows:

$$
W(q, r, t)=\exp \left\{-\frac{d(q, r)}{t+1}\right\}
$$

where $q, r \in X$, and $t>0$. Clearly, $W(q, r, t)$ satisfies the conditions of (GV1)-(GV3) and (GV5).

Moreover, for all $q, r, l \in X$ and $t, s>0$, it is clear that

$$
\begin{aligned}
W(q, l, t+s) & =\exp \left\{-\frac{d(q, l)}{t+s+1}\right\} \\
& \geq \exp \left\{-\frac{d(q, r)+d(r, l)}{t+s+1}\right\} \\
& \geq \exp \left\{-\frac{d(q, r)}{t+1}\right\} \cdot \exp \left\{-\frac{d(r, l)}{t+1}\right\} \\
& =W(q, r, t) \cdot W(r, l, s),
\end{aligned}
$$

that is, Condition (GV4) holds.

Let $\phi(x)=\frac{1}{4} x(x \in X), F(y)=-\frac{1}{\ln y}(0<y<1)$ and $\tau=\frac{1}{2}$. Since

$$
\begin{aligned}
\tau \cdot F(W(\phi(q), \phi(r), t)) & =-\tau \cdot \frac{1}{\ln \left[\exp \left\{-\frac{1}{4} \frac{d(q, r)}{t+1}\right\}\right]}=4 \tau \cdot \frac{t+1}{d(q, r)}=2 \cdot \frac{t+1}{d(q, r)} \\
& >\frac{t+1}{d(q, r)}=F(W(q, r, t))
\end{aligned}
$$

holds for all $q, r \in X, q \neq r$ and $t>0$, then Condition (14) is fulfilled. Hence, by Theorem 1, it follows that $\phi$ has a unique fixed point. It is worth mentioning that this example is true for arbitrary function $\phi(q)=k q$, where $0<k<1$ is a constant with $\tau>k$.

The following example shows our contractive condition (14) which greatly generalizes the contractive condition of [22]. 
Example 2. Let $(X, d)$ be a metric space and $T$ a $t$-norm. Then for all $q, r \in X$ and $t>0$,

$$
W(q, r, t)=\frac{t+1}{t+1+d(q, r)}
$$

defines a fuzzy set.

Define a function $F(x)=x$ on $[0,1]$ and let $\tau \in(0,1)$ be a constant. If Condition (14) is fulfilled, then

$$
\begin{aligned}
W(\phi(q), \phi(r), t) & =F(W(\phi(q), \phi(r), t)) \\
& \geq \frac{1}{\tau} F(W(q, r, t))=\frac{t+1}{\tau \cdot(t+1+d(q, r))}=\frac{\frac{t}{\tau}+\frac{1}{\tau}}{t+1+d(q, r)} \\
& \geq \frac{\frac{t}{\tau}+1}{\frac{t}{\tau}+1+d(q, r)}=W\left(q, r, \frac{t}{\tau}\right)
\end{aligned}
$$

holds for all $q, r \in X$ and $t>0$. That is to say, we obtain the contractive condition (1) from [22].

Theorem 2. Let $(X, W, T)$ be a complete fuzzy metric space and $F \in \mathcal{F}$ be a continuous mapping. If $\phi: X \rightarrow X$ is a fuzzy F-contraction, then $\phi$ has a unique fixed-point in $X$.

Proof. Choose $q_{0} \in X$ and define a sequence $\left\{q_{n}\right\}$ by $q_{n+1}=\phi\left(q_{n}\right)\left(n \in \mathbb{N}_{0}\right)$. If $q_{n}=q_{n+1}$ for some $n \in \mathbb{N}_{0}$, then the proof is finished. Assume that $q_{n} \neq q_{n+1}$ for any $n \in \mathbb{N}_{0}$. From the definition of the F-contraction, we have

$$
F\left(W\left(q_{n+1}, q_{m+1}, t\right)\right)>\tau \cdot F\left(W\left(q_{n+1}, q_{m+1}, t\right)\right) \geq F\left(W\left(q_{n}, q_{m}, t\right)\right),
$$

then $W\left(q_{n+1}, q_{m+1}, t\right)>W\left(q_{n}, q_{m}, t\right)$ for any $n>m$. Let $a_{m}(t)=\inf _{n>m} W\left(q_{n}, q_{m}, t\right)$. Notice that

$$
\inf _{n>m} W\left(q_{n+1}, q_{m+1}, t\right) \geq \inf _{n>m} W\left(q_{n}, q_{m}, t\right),
$$

then $a_{m+1}(t) \geq a_{m}(t)$, for any $m \in \mathbb{N}$. Since $\left\{a_{m}(t)\right\}$ is bounded, then there exists $a(t) \in$ $[0,1]$ such that $\lim _{m \rightarrow \infty} a_{m}(t)=a(t)$ for all $t>0$. Let us prove that $a(t)=1$ for all $t>0$. Suppose the contrary, and there exists $s>0$ such that $0 \leq a(s)<1$. Then by (14), for some $\tau \in(0,1)$, one has

$$
\begin{aligned}
\lim _{m \rightarrow \infty} F\left(\inf _{n>m} W\left(q_{n+1}, q_{m+1}, s\right)\right) & \geq \tau \cdot \lim _{m \rightarrow \infty} F\left(\inf _{n>m} W\left(q_{n+1}, q_{m+1}, s\right)\right) \\
& \geq \lim _{m \rightarrow \infty} F\left(\inf _{n>m} W\left(q_{n}, q_{m}, s\right)\right) .
\end{aligned}
$$

Using the assumption that $F$ is continuous, we have

$$
F(a(s)) \geq \tau \cdot F(a(s)) \geq F(a(s)),
$$

which means that $F(a(s))=0$. This is in contradiction with $F(a(s))>0$. Thus,

$$
\lim _{m \rightarrow \infty} \inf _{n>m} W\left(q_{n}, q_{m}, t\right)=1
$$

for any $t>0$. Consequently,

$$
\lim _{m, n \rightarrow \infty} W\left(q_{n}, q_{m}, t\right)=1
$$

for any $t>0$. Thus, $\left\{q_{n}\right\}$ is a Cauchy sequence. Since $(X, W, T)$ is complete, then there exists $q \in X$ such that $\lim _{n \rightarrow \infty} q_{n}=q$.

Taking advantage of (14), we have

$$
F\left(W\left(q_{n+1}, \phi(q), t\right)\right)>\tau \cdot F\left(W\left(q_{n+1}, \phi(q), t\right)\right) \geq F\left(W\left(q_{n}, q, t\right)\right)
$$


for all $n \in \mathbb{N}$. Letting $n \rightarrow \infty$ and using the assumption that $F$ is continuous, we have

$$
F(W(q, \phi(q), t)) \geq \tau \cdot F(W(q, \phi(q), t)) \geq F(1) .
$$

Thus, it leads to $W(q, \phi(q), t) \geq 1$. Therefore, $q=\phi(q)$.

Suppose now that $\phi$ has distinct fixed-points $q, r \in X$, then by (14), we obtain

$$
F(W(q, r, t))=F(W(\phi(q), \phi(r), t))>\tau \cdot F(W(\phi(q), \phi(r), t)) \geq F(W(q, r, t)) .
$$

This is a contradiction. Hence, $q=r$.

Theorem 3. Let $(X, W, T)$ be a complete fuzzy metric space such that $\lim _{t \rightarrow 0^{+}} W(q, r, t)>0$ for all $q, r \in X$. Let $\phi: X \rightarrow X$ be a mapping and $F \in \mathcal{F}$. Suppose that for all $q, r \in X, q \neq r$ and $t>0$, there exists $\tau \in(0,1)$ such that

$$
\tau \cdot F(W(\phi(q), \phi(r), t)) \geq F(\min \{W(q, r, t), W(q, \phi(q), t), W(r, \phi(r), t)\}) .
$$

Then, $\phi$ has a unique fixed point, provided that $\phi$ or $F$ is continuous.

Proof. Choose $q_{0} \in X$ and define a sequence $\left\{q_{n}\right\}$ as follows: $q_{n+1}=\phi\left(q_{n}\right)\left(n \in \mathbb{N}_{0}\right)$. By (21), we have

$$
\begin{aligned}
F\left(W\left(q_{n}, q_{n+1}, t\right)\right) & =F\left(W\left(\phi\left(q_{n-1}\right), \phi\left(q_{n}\right), t\right)\right) \\
& >\tau \cdot F\left(W\left(\phi\left(q_{n-1}\right), \phi\left(q_{n}\right), t\right)\right) \\
& \geq F\left(\min \left\{W\left(q_{n-1}, q_{n}, t\right), W\left(q_{n-1}, q_{n}, t\right), W\left(q_{n}, q_{n+1}, t\right)\right\}\right) \\
& =F\left(\min \left\{W\left(q_{n-1}, q_{n}, t\right), W\left(q_{n}, q_{n+1}, t\right)\right\}\right),
\end{aligned}
$$

for all $n \in \mathbb{N}$ and $t>0$.

If $\min \left\{W\left(q_{n-1}, q_{n}, t\right), W\left(q_{n}, q_{n+1}, t\right)\right\}=W\left(q_{n}, q_{n+1}, t\right)$, then by (22), we get

$$
W\left(q_{n}, q_{n+1}, t\right)>W\left(q_{n}, q_{n+1}, t\right),
$$

which is a contradiction. If $\min \left\{W\left(q_{n-1}, q_{n}, t\right), W\left(q_{n}, q_{n+1}, t\right)\right\}=W\left(q_{n-1}, q_{n}, t\right)$, then by (22), we have

$$
W\left(q_{n}, q_{n+1}, t\right)>W\left(q_{n-1}, q_{n}, t\right) .
$$

Following the proof of Theorem 1, we find $q^{*} \in X$ such that $\lim _{n \rightarrow \infty} q_{n}=q^{*}$.

Suppose first that $\phi$ is continuous. Then, by the construction of sequence $\left\{q_{n}\right\}$, it follows that $\phi$ has a fixed point $q^{*}$.

Suppose that $F$ is continuous. Then, by (21), we have

$$
\begin{aligned}
F\left(W\left(q_{n+1}, \phi\left(q^{*}\right), t\right)\right) & >\tau \cdot F\left(W\left(q_{n+1}, \phi\left(q^{*}\right), t\right)\right) \\
& \geq F\left(\min \left\{W\left(q_{n}, q^{*}, t\right), W\left(q_{n}, q_{n+1}, t\right), W\left(q^{*}, \phi\left(q^{*}\right), t\right)\right\}\right),
\end{aligned}
$$

for all $n \in \mathbb{N}$ and $t>0$. If $\phi\left(q^{*}\right) \neq q^{*}$, then taking $n \rightarrow \infty$ from both sides of (23), we have $F\left(W\left(q^{*}, \phi\left(q^{*}\right), t\right)\right) \geq \tau \cdot F\left(W\left(q^{*}, \phi\left(q^{*}\right), t\right)\right) \geq F\left(\min \left\{1,1, W\left(q^{*}, \phi\left(q^{*}\right), t\right)\right\}\right)=F\left(W\left(q^{*}, \phi\left(q^{*}\right), t\right)\right)$, which means that $F\left(W\left(q^{*}, \phi\left(q^{*}\right), t\right)\right)=0$. This is in contradiction with $F\left(W\left(q^{*}, \phi\left(q^{*}\right), t\right)\right)>0$.

Finally, we prove the uniqueness of the fixed point. Assume that $\phi$ has two distinct fixed points, $p^{*}, q^{*}$. Then, by (21), we have

$$
\begin{aligned}
F\left(W\left(p^{*}, q^{*}, t\right)\right) & =F\left(W\left(\phi\left(p^{*}\right), \phi\left(q^{*}\right), t\right)\right) \\
& >\tau \cdot F\left(W\left(\phi\left(p^{*}\right), \phi\left(q^{*}\right), t\right)\right) \\
& \geq F\left(\min \left\{W\left(p^{*}, q^{*}, t\right), W\left(p^{*}, \phi\left(p^{*}\right), t\right), W\left(q^{*}, \phi\left(q^{*}\right), t\right)\right\}\right)
\end{aligned}
$$




$$
\begin{aligned}
& =F\left(\min \left\{W\left(p^{*}, q^{*}, t\right), W\left(p^{*}, p^{*}, t\right), W\left(q^{*}, q^{*}, t\right)\right\}\right) \\
& =F\left(\min \left\{W\left(p^{*}, q^{*}, t\right), 1,1\right\}\right) \\
& =F\left(W\left(p^{*}, q^{*}, t\right)\right),
\end{aligned}
$$

which is a contradiction. Therefore, $p^{*}=q^{*}$.

Author Contributions: H.H. designed the research and wrote the paper. B.C. and T.D. offered the draft preparation and gave the methodology, D.R. and M.B. co-wrote and made revisions to the paper. H.H. and T.D. gave the support of funding acquisition. All authors have read and agreed to the published version of the manuscript.

Funding: The first author acknowledges the financial support from the Natural Science Foundation of Chongqing of China (No. cstc2020jcyj-msxmX0762), and the Initial Funding of Scientific Research for High-level Talents of Chongqing Three Gorges University of China (No. 2104/09926601). The third author acknowledges the financial support from the Ministry of Education, Science and Technological Development of the Republic of Serbia (No. 451-03-68/2020-14/200134).

Institutional Review Board Statement: Not applicable.

Informed Consent Statement: Not applicable.

Data Availability Statement: The data presented in this study are available upon request from the corresponding author.

Acknowledgments: The authors thank the editor and the referees for their valuable comments and suggestions which improved greatly the quality of this paper.

Conflicts of Interest: The authors declare no conflict of interest.

\section{References}

1. Zadeh, L.A. Fuzzy sets. Inf. Control 1965, 8, 338-353. [CrossRef]

2. George, A.; Veeramani, P. On some results in fuzzy metric spaces. Fuzzy Sets Syst. 1994, 64, 395-399. [CrossRef]

3. Schweizer B.; Sklar A. Statistical metric spaces. Pac. J. Math. 1960, 10, 314-334. [CrossRef]

4. Banach, S. Sur les opérations dans les ensembles abstraits et leur application aux éuations intégrales. Fund. Math. 1922, 3, 133-181. [CrossRef]

5. Grabiec, M. fixed-points in fuzzy metric spaces. Fuzzy Sets Syst. 1988, 27, 385-389. [CrossRef]

6. Gregori, V.; Miñana, J.-J. On fuzzy $\psi$-contractive sequences and fixed-point theorems. Fuzzy Sets Syst. 2016, 300, 93-101. [CrossRef]

7. Gregori, V.; Miñana, J.-J. Some remarks on fuzzy contractive mappings. Fuzzy Sets Syst. 2014, 251, 101-103. [CrossRef]

8. Gregori, V.; Sapena, A. On fixed-point theorems in fuzzy metric spaces. Fuzzy Sets Syst. 2002, 125, 245-252. [CrossRef]

9. Mihet D. Erratum to " Fuzzy $\psi$-contractive mappings in non-Archimedean fuzzy metric spaces [Fuzzy Sets and Systems. 159 (2008), 739-744]". Fuzzy Sets Syst. 2010, 161, 1150-1151. [CrossRef]

10. Mihet, D. Fuzzy $\psi$-contractive mappings in non-Archimedean fuzzy metric spaces. Fuzzy Sets Syst. 2008, 159, 739-744. [CrossRef]

11. Gregori, V.; Miñana, J.-J.; Miravet, D. Contractive sequences in fuzzy metric spaces. Fuzzy Sets Syst. 2020, 379, 125-133. [CrossRef]

12. Wardowski, D. Fuzzy contractive mappings and fixed-points in fuzzy metric space. Fuzzy Sets Syst. 2013, 222, 108-114. [CrossRef]

13. Wardowski, D. Fixed points of a new type of contractive mappings in complete metric spaces. Fixed Point Theory Appl. 2012, 2012, 94. [CrossRef]

14. Shukla, S.; Gopal, D.; Sintunavarat, W. A new class of fuzzy contractive mappings and fixed-point theorems. Fuzzy Sets Syst. 2018, 350, 85-94. [CrossRef]

15. Secelean, N.A. Iterated function system consisting of F-contractions. Fixed Point Theory Appl. 2013, 2013, 277. [CrossRef]

16. Gregori, V.; Morillas, S.; Sapena, A. On a class of compatible fuzzy metric spaces. Fuzzy Sets Syst. 2010, 161, 2193-2205. [CrossRef]

17. Rodriguez-Lopez, J.; Romaguera, S. The Hausdorff fuzzy metric on compact sets. Fuzzy Sets Syst. 2004, 147, 273-283. [CrossRef]

18. Tirado, P. Contraction mappings in fuzzy quasi-metric spaces and [0; 1]-fuzzy posets. Fixed Point Theory 2012, 13, $273-283$.

19. Radenović, S.; Vetro, F.; Vujaković, J. An alternative and easy approach to fixed-point results via simulation functions. Demonstr. Math. 2017, 50, 223-230. [CrossRef]

20. Chandok, S.; Huang, H.; Radenović, S. Some fixed-point results for the generalized F-suzuki type contractions in $b$-metric spaces. Sahand Commun. Math. Anal. 2018, 11, 81-89.

21. Secelean, N.A.; Mathew, S.; Wardowski, D. New fixed-point results in quasi-metric spaces and applications in fractals theory. Adv. Differ. Equ. 2019, 2019, 177. [CrossRef]

22. Sehgal, V.M.; Bharucha-Reid, A.T. Fixed points of contaction mappings on probabilistic metric spaces. Math. Syst. Theory 1972, 6, 97-102. [CrossRef] 